\title{
Synaptic Microtubule-Associated Protein EB3 and SRC Phosphorylation Mediate Structural and Behavioral Adaptations During Withdrawal From Cocaine Self-Administration
}

\author{
Erin S. Calipari, ${ }^{1 \star}{ }^{\circledR}$ Arthur Godino, ${ }^{1 \star}$ Marine Salery, ${ }^{1}$ Diane M. Damez-Werno, ${ }^{1}$ Michael E. Cahill, ${ }^{1}$ CCraig T. Werner, ${ }^{2}$ \\ Amy M. Gancarz, ${ }^{2}$ Emily G. Peck, ${ }^{1}$ Zahra Jlayer, ${ }^{1}$ Jacqui Rabkin, ${ }^{1}$ Joseph A. Landry, ${ }^{1}$ Alexander C.W. Smith, ${ }^{1}$ \\ Paola Defilippi, ${ }^{3}$ Paul J. Kenny, ${ }^{1}$ Yasmin L. Hurd, ${ }^{1}$ Rachael L. Neve, ${ }^{4}$ David M. Dietz, ${ }^{2}$ and $\odot$ Eric J. Nestler ${ }^{1}$ \\ ${ }^{1}$ Department of Neuroscience and Friedman Brain Institute, Icahn School of Medicine at Mount Sinai, New York, New York 10029, ${ }^{2}$ Department of \\ Pharmacology and Toxicology, Jacobs School of Medicine and Biomedical Sciences, University at Buffalo, Buffalo, New York 14260 , ${ }^{3}$ Molecular \\ Biotechnology Center, Departments of Molecular Biotechnology and Health Sciences and of Neuroscience, University of Torino, 10126 Torino, Italy, \\ and ${ }^{4}$ Gene Delivery Technology Core, Massachusetts General Hospital, Boston, Massachusetts 02114
}

Addictive behaviors, including relapse, are thought to depend in part on long-lasting drug-induced adaptations in dendritic spine signaling and morphology in the nucleus accumbens (NAc). While the influence of activity-dependent actin remodeling in these phenomena has been studied extensively, the role of microtubules and associated proteins remains poorly understood. We report that pharmacological inhibition of microtubule polymerization in the NAc inhibited locomotor sensitization to cocaine and contextual reward learning. We then investigated the roles of microtubule end-binding protein 3 (EB3) and SRC kinase in the neuronal and behavioral responses to volitionally administered cocaine. In synaptoneurosomal fractions from the NAc of self-administering male rats, the phosphorylation of SRC at an activating site was induced after $1 \mathrm{~d}$ of withdrawal, while EB3 levels were increased only after $30 \mathrm{~d}$ of withdrawal. Blocking SRC phosphorylation during early withdrawal by virally overexpressing SRCIN1, a negative regulator of SRC activity known to interact with EB3, abolished the incubation of cocaine craving in both male and female rats. Conversely, mimicking the EB3 increase observed after prolonged withdrawal increased the motivation to consume cocaine in male rats. In mice, the overexpression of either EB3 or SRCIN1 increased dendritic spine density and altered the spine morphology of NAc medium spiny neurons. Finally, a cocaine challenge after prolonged withdrawal recapitulated most of the synaptic protein expression profiles observed at early withdrawal. These findings suggest that microtubule-associated signaling proteins such as EB3 cooperate with actin remodeling pathways, notably SRC kinase activity, to establish and maintain long-lasting cellular and behavioral alterations following cocaine self-administration.

Key words: addiction; dendritic spines; MAPRE3; nucleus accumbens; podophyllotoxin; SRCIN1

Significance Statement

Drug-induced morphological restructuring of dendritic spines of nucleus accumbens neurons is thought to be one of the cellular substrates of long-lasting drug-associated memories. The molecular basis of these persistent changes has remained incompletely understood. Here we implicate for the first time microtubule function in this process, together with key players such as microtubule-bound protein EB3 and synaptic SRC phosphorylation. We propose that microtubule and actin remodeling cooperate during withdrawal to maintain the plastic structural changes initially established by cocaine self-administration. This work opens new translational avenues for further characterization of microtubule-associated regulatory molecules as putative drug targets to tackle relapse to drug taking.

\section{Introduction}

Drug addiction results from dysfunction within the reward circuitry of the brain (Koob and Volkow, 2010), in which excitatory projections to the nucleus accumbens (NAc) play an integral role in the acquisition and execution of goal-oriented behaviors and reward learning (Day and Carelli, 2007). Drug-related reward 
learning and seeking mechanisms are similar to those of natural rewards (Kelley and Berridge, 2002); however, only drug-seeking increases - or "incubates"-with prolonged periods of withdrawal (Krasnova et al., 2014). This incubation of drug seeking is considered a key mediator of relapse that occurs following long abstinence periods in both human addicts and rodent models of drug addiction (Wolf, 2016). Mechanistically, drugs of abuse, such as cocaine, mimic reward-related learning signals by increasing extracellular dopamine in the NAc to levels far exceeding those of natural reinforcers (Di Chiara and Imperato, 1988). This results in maladaptive long-term changes in NAc neuronal function that underlie pathological reward learning and ultimately drug seeking and relapse (Volkow and Morales, 2015).

Excitatory afferents synapse primarily on dendritic spines (Nimchinsky et al., 2002). Consequent adaptations in postsynaptic spine structure mediate many of the changes in synaptic transmission that drive learning (Lamprecht and LeDoux, 2004) and addictive behaviors (Russo et al., 2010; Toda et al., 2010; Huang et al., 2015), as alterations in dendritic spine number and morphology in the NAc occur after exposure to nearly every drug of abuse (Robinson and Kolb, 2004). At the molecular level, synaptic activity triggers diverse signaling pathways, which in turn regulate cytoskeleton-associated proteins and cytoskeletal reorganization, ultimately leading to spine growth, retraction, or morphological changes (Borovac et al., 2018). However, studies of activity-dependent structural plasticity have focused predominantly on actin cytoskeleton dynamics (Hotulainen and Hoogenraad, 2010), and it remains unclear how newly formed thin, immature spines are stabilized into mushroom-shaped, stable spines (Kasai et al., 2003) that can persist even after prolonged drug withdrawal.

We hypothesize that microtubule dynamics, in conjunction with actin remodeling, contribute to this process. Microtubules are polarized tubules assembled from $\alpha / \beta$-tubulin heterodimers. The plus-end of microtubules drives polymerization and growth, while the minus-end drives microtubule "catastrophe" (Geraldo and Gordon-Weeks, 2009). Emerging evidence implicates microtubule dynamics in dendritic spine architecture and activity-dependent remodeling. Growth from the plus-end of microtubules into dendritic spines of cultured cortical and hippocampal neurons is triggered by neuronal activation (Hu et al., 2008), and this rapid invasion is responsible for later spine stabilization (Jaworski et al., 2009). Several classes of microtubulebinding proteins regulate their dynamics (Coles and Bradke, 2015). End-binding protein 3 (EB3), also known as microtubuleassociated protein RP/EB family member 3 (MAPRE3), binds microtubule plus-ends to promote mature mushroom spines over immature thin spines in cultured neurons (Jaworski et al., 2009). Interestingly, EB3 interacts with SRCIN1/p140Cap (Jaworski et al., 2009), a negative regulator of SRC kinase (Di Stefano et al., 2007) previously implicated in structural plasticity and learning (Repetto et al., 2014), as well as cocaine-induced adap-

Research Foundation (E.S.C.), the Whitehall Foundation (E.S.C.), and the Edward Mallinckrodt Jr. Foundation (E.S.C.).

*E.S.C. and A.G. contributed equally to this work.

The authors declare no competing financial interests.

Correspondence should be addressed to Eric J. Nestler at eric.nestler@mssm.edu.

E. S. Calipari's present address: Department of Pharmacology, Vanderbilt Center for Addiction Research, Vanderbilt University School of Medicine, Nashville, TN 37235.

M. E. Cahill's present address: Department of Comparative Biosciences, School of Veterinary Medicine, University of Wisconsin, Madison, WI 53706.

https://doi.org/10.1523/JNEUROSCI.0024-19.2019

Copyright $\odot 2019$ the authors tations (Damez-Werno et al., 2016). Cooperation between EB3 and SRC activity via SRCIN1 has been proposed to mediate cross talk between actin and microtubule dynamics that are crucial for synaptic plasticity in vitro (Jaworski et al., 2009); however, the contribution of such a mechanism to complex in vivo behaviors has yet to be determined.

Here, we examine the role of microtubules, microtubuleassociated EB3, SRCIN1, and SRC in a rodent model of cocaine addiction. We first demonstrate that microtubules are involved in cocaine-induced plasticity. We then show that SRC phosphorylation and EB3 levels are increased at the synapse after early and/or late withdrawal from cocaine self-administration. We next explore the behavioral and structural correlates of these changes, and establish that SRC phosphorylation is necessary for the incubation of cocaine craving, EB3 modulates the motivation to seek cocaine, and both are associated with alterations in spine density and morphology. Together, these results implicate for the first time microtubule-related signaling events in cocaineinduced molecular, structural, and behavioral adaptations, providing new insight into the pathophysiology of addiction.

\section{Materials and Methods}

Animals. Animals were housed in the animal facilities at Mount Sinai or at the University at Buffalo. Male C57BL/6J mice (8 weeks old; weight, $20-25 \mathrm{~g}$; The Jackson Laboratory) were maintained on a $12 \mathrm{~h}$ light/dark cycle (7:00 A.M. lights on; 7:00 P.M. lights off) and were provided with food and water ad libitum. For self-administration experiments, male (weight, 300-375 g) and female (weight, 250-325 g) rats were maintained on a $12 \mathrm{~h}$ reverse light/dark cycle (7:00 A.M. lights off; 7:00 P.M. lights on) and restricted to $95 \%$ of free-feeding body weight to improve consistency of the self-administration behavior. All animals were maintained according to the National Institutes of Health Guidelines for Association for Assessment and Accreditation of Laboratory Animal Care accredited facilities. All experimental protocols were approved by the Institutional Animal Care and Use Committee at Mount Sinai.

Conditioned place preference. An unbiased conditioned place preference assay was carried out in male mice as described previously (Calipari et al., 2018) using three conditioning sessions with a dose of $10 \mathrm{mg} / \mathrm{kg}$, i.p., cocaine.

Microinfusion experiments. Mice were surgically implanted with bilateral guide cannula (Plastics One) as described previously (Calipari et al., 2018). The following coordinates were used to target the NAc bilaterally: from bregma, anteroposterior $+1.5 \mathrm{~mm}$, mediolateral $+1.0 \mathrm{~mm}$, and dorsoventral $-4.3 \mathrm{~mm}$. The infusion probes were designed to protrude $200 \mu \mathrm{m}$ below the tip of the guide cannula so that the drug was injected directly into the NAc at $-4.5 \mathrm{~mm}$ bregma. Approximately $30 \mathrm{~min}$ before daily conditioning sessions, animals received bilateral intra-NAc infusions of $100 \mu \mathrm{M}$ podophyllotoxin diluted in a vehicle composed of artificial CSF solution (Calipari et al., 2018) containing 0.5\% DMSO, or the vehicle solution only at a continuous rate of $0.1 \mu \mathrm{l} / \mathrm{min}$ via a microinfusion pump with a total injection volume of $0.5 \mu \mathrm{l}$ (Harvard Apparatus). Injector needles remained in place for $5 \mathrm{~min}$ before being removed. Mice were left undisturbed for $\pm 5 \mathrm{~min}$ before the conditioning. The concentration of podophyllotoxin used was based on published studies (Jordan et al., 1992).

Self-administration surgery and training. Rats were implanted with indwelling jugular catheters, as described previously (Calipari et al., 2018). Following 2-4 d of recovery, animals were placed into operant drugconditioning chambers (Med Associates) for $6 \mathrm{~h}$ daily and were allowed to acquire cocaine self-administration on a fixed ratio one (FR1) schedule of reinforcement using a cocaine dose of $0.8 \mathrm{mg} / \mathrm{kg} /$ infusion delivered over $5 \mathrm{~s}$, as described previously (Calipari et al., 2018). Acquisition was defined as the first session in which an animal allocated $>70 \%$ of their responses to the active lever, and when a stable pattern of interinfusion intervals was present. Rats underwent $10 \mathrm{~d}$ of self-administration and were then killed after 1 or $30 \mathrm{~d}$ of withdrawal, with or without an intraperitoneal cocaine challenge for subsequent biochemical analyses. For 
the incubation of a craving experiment, rats also remained on an FR1 schedule for $10 \mathrm{~d}$ before being injected intra-NAc with herpes simplex virus (HSV) vectors and tested for drug seeking (see below). For a select set of experiments, animals went through a within-session threshold procedure, which is described in detail below. Before the threshold procedure, rats were trained on a short-access (2-h-long sessions) FR1 until they met acquisition criteria and were then injected with HSVs. For all experiments, rats that failed to reach acquisition or maintain selfadministration behavior were excluded from further analysis $(<10 \%$ of total).

HSV surgeries. Mice and rats were anesthetized with ketamine (100 $\mathrm{mg} / \mathrm{kg}$, i.p.) and xylazine $(10 \mathrm{mg} / \mathrm{kg}$, i.p.) and prepared for stereotaxic surgery. Thirty-three gauge syringe needles (Hamilton) were used to bilaterally infuse at a $0.1 \mu \mathrm{l} / \mathrm{min}$ flow rate either $0.5 \mu \mathrm{l}$ (mice) or $0.7 \mu \mathrm{l}$ (rats) of bicistronic p1005 HSVs expressing GFP alone or GFP plus either EB3 or SRCIN1 into the NAc. Coordinates for rats were as follows: from bregma, anteroposterior $+1.9 \mathrm{~mm}$, mediolateral $+2.8 \mathrm{~mm}$, and dorsoventral $-7.1 \mathrm{~mm}$; angle, $7^{\circ}$ ). Coordinates for mice were as follows: from bregma, anteroposterior $+1.5 \mathrm{~mm}$, mediolateral $+1.6 \mathrm{~mm}$, and dorsoventral $-4.5 \mathrm{~mm}$; angle, $10^{\circ}$ ). Animals with impeded recovery from surgery were discarded from further behavioral testing $(<15 \%$ of the total).

Incubation of craving. After $10 \mathrm{~d}$ of cocaine self-administration training and HSV injection, rats (both males and females) were tested for cue seeking after either 3 or $30 \mathrm{~d}$ of withdrawal. Rats were placed in the self-administration box for $30 \mathrm{~min}$. Active lever presses-nonretracting-resulted in the presentation of the conditioned reinforcing cue light that previously confirmed cocaine delivery, but no cocaine was available. The number of active lever presses in these conditions is commonly used as a measure of drug-seeking behavior, and is known to increase over withdrawal time- a process termed incubation (Grimm et al., 2001). No differences between male and female rats were noted at any level; thus, data from both sexes were averaged and analyzed together.

Threshold procedure. Following acquisition of cocaine self-administration (short-access) and HSV injection, male rats underwent the threshold procedure to determine differences in consummatory and appetitive responding using previously published behavioral economics methods (Oleson and Roberts, 2009; Calipari et al., 2018). Briefly, a descending series of 11-unit doses of cocaine $(421,237,133,75,41,24$, $13,7.5,4.1,2.4$, and $1.3 \mu \mathrm{g} /$ injection) are available upon an FR1 schedule with no timeouts, and levers remain extended. Consequently, the "price" of cocaine - the number of responses required to obtain $1 \mathrm{mg}$-increases over time. Both the number of lever presses and the total cocaine intake are measured for each price bin and plotted as a function of price. The resulting demand curves can be used for mathematical curve fitting using the following equation: $\log _{10}(Q)=\log _{10}\left(Q_{0}\right)+k\left(e^{-\alpha Q_{0} C-1}\right)$, where $Q$ is consumption and $C$ is the varying cost of the reinforcer (Hursh and Silberberg, 2008). The value $k$ was set to the average of all $k$ values obtained after a first round of individual curve fitting, to which 0.5 was added, and was equal to 2.20. The parameters $\alpha, Q_{0}$, and $P_{\max }$ were then calculated for each animal. $Q_{0}$ is a measure of the preferred level of cocaine consumption of the animals and was measured as the amount of consumption, in milligrams, at a minimally constraining price. $\mathrm{P}_{\max }$ is the price at which the animal no longer emits enough responses to maintain the $Q_{0}$ level of intake and consumption decreases. It is the inflection point of the demand curve and can be measured as the point for which the first derivative slope is equal to -1 . The measure of elasticity $\alpha$, also termed the essential value, is a measure of how sensitive the demand is to price. Thus, if elasticity is high, as price increases, responding will drop off quickly. If the behavior is inelastic, animals will be motivated to continue consuming drug, regardless of cost.

Subcellular fractionation. Rat tissue was collected via decapitation followed by rapid bilateral NAc punch dissections from 1-mm-thick coronal brain sections using a 12 gauge needle and frozen on dry ice. Samples were fractionated into P1 (crude nuclear), S2 (cytoplasm), and P2 (crude synaptoneurosomal) components using our established procedures $(\mathrm{Ca}-$ hill et al., 2016). Briefly, unpooled NAc samples were homogenized in $150 \mu$ l of HEPES-buffered sucrose ( $0.32 \mathrm{~m}$ sucrose, 4 mM HEPES, $\mathrm{pH} 7.4$ ) complemented with protease and serine/threonine and tyrosine phos- phatase inhibitors before successive centrifugation and wash steps. Final pellets were resuspended in RIPA buffer, $\mathrm{pH}$ 7.4, complemented with protease and serine/threonine and tyrosine phosphatase inhibitors. Protein concentrations of synaptoneurosomal (P2) fractions were determined using a $D C$ Protein Assay (Bio-Rad).

Western blotting and antibodies. SDS-PAGE protein separation and Western blotting were performed according to manufacturer instructions and our published protocols (Cahill et al., 2016). Equal amounts of $\mathrm{P} 2$ proteins were mixed with SDS- and DTT-containing reducing buffer (Thermo Fisher Scientific), heated to $95^{\circ} \mathrm{C}$ for 5 min before being separated by SDS-PAGE with Criterion Precast Gels (4-15\% Tris-HCl; BioRad), and transferred onto PVDF Immobilon-P $0.45 \mu \mathrm{m}$ membranes. Membranes were blocked in Tris-buffered saline containing $5 \%$ bovine serum albumin and $0.1 \%$ Tween-20 for $1 \mathrm{~h}$ at room temperature. The following primary antibodies were diluted at 1:1000 (unless otherwise indicated) in blocking solution and incubated overnight at $4^{\circ} \mathrm{C}$ : actin (mouse; 1:40,000; catalog \#691002, MP Biomedicals); EB3 (rabbit; cata$\log \#$ ab157217, Abcam); $\beta$-tubulin (rabbit, catalog \#2146S, Cell Signaling Technology); GluN2B (rabbit; catalog \#06-600, Millipore); PSD-95 (rabbit; catalog \#ab18258, Abcam); SRC (rabbit; catalog \#2123S, Cell Signaling Technology), and SRC family Y416 (rabbit; catalog \#6943S, Cell Signaling Technology). This latter antibody recognizes the phosphorylated forms of several SRC family members. Moreover, because SRC and FYN migrate similarly on Western blots, the antibody cannot distinguish between phospho-SRC and phospho-FYN. We also used an anti-SRCIN1 antibody that has been validated previously (Repetto et al., 2014; Damez-Werno et al., 2016). After washing, membranes were incubated with anti-mouse (catalog \#PI-2000, Vector Laboratories) or antirabbit (catalog \#PI-1000, Vector Laboratories) peroxidase-conjugated secondary antibodies diluted 1:50,000 in blocking solution for $2 \mathrm{~h}$, washed thoroughly, and developed using SuperSignal West Dura Substrate (Thermo Fisher Scientific). Quantification was performed by densitometry using ImageJ (U.S. National Institutes of Health). Protein levels were normalized to actin or to total SRC for the phosphorylated antibody. Between primary antibodies, membranes were stripped using Restore Plus Stripping Buffer (Thermo Fisher Scientific).

Spine imaging and counting. Dendritic spines from HSV-infected neurons were imaged and analyzed using established procedures (Cahill et al., 2016). Briefly, brains were perfused and postfixed using $4 \%$ paraformaldehyde, then sectioned at $100 \mu \mathrm{m}$. Native GFP signal was enhanced by immunohistochemistry using an anti-GFP primary antibody (chicken; 1:500; catalog \#GFP-1020, Aves Labs) and the Alexa Fluor 488 antichicken secondary antibody (1:500; Jackson ImmunoResearch) before imaging on an LSM 710 Confocal Microscope (Carl Zeiss) using a 100X objective lens. After deconvolution, dendrite images were analyzed using NeuronStudio (http://research.mssm.edu/cnic/tools-ns.html). Spines were classified using the following criteria. Thin spines have a neck ratio value (head to neck diameter ratio) of $<1.1$ and a length to spine head diameter of $>2.0$. Mushroom spines have a neck ratio value of $>1.1$ and a spine head diameter of $\geq 0.3 \mu \mathrm{m}$. Stubby spines were not estimated. All imaging and quantification were performed by an investigator blind to experimental conditions.

$R N A$ extraction and quantitative real-time PCR. Mouse tissue was collected via cervical dislocation followed by rapid dissection of bilateral NAc from 1-mm-thick coronal brain sections using a 14 gauge needle and frozen on dry ice. Samples were subsequently homogenized in TRIzol (Thermo Fisher Scientific), and RNA extraction was performed using RNeasy Micro columns (Qiagen) according to manufacturer instructions. RNA 260/280 ratios between 1.8 and 2 were confirmed using spectroscopy, and reverse transcription was achieved using the iScript kit (Bio-Rad). Quantitative PCR using SYBR-Green (Quanta) was used to quantify cDNA using an Applied Biosystems 7900HT RT-PCR system. Each reaction was performed in triplicate and analyzed using the $\Delta \Delta \mathrm{C}(\mathrm{t})$ method with Gapdh as the normalization control. Primer sequences are listed below, from $5^{\prime}$ to $3^{\prime}$, as follows: mouse EB3: forward, TAGCAC CACCTCCTAACCCA; reverse GCAGCTGCTGGTTGAGTT; mouse SRCIN1: forward, CCTCGGCCATCAAGGATGAA; reverse, TGGGC CTGGATCCTCATGTA; mouse SRC: forward, CACTGTCACCTC CCCACAAA; reverse, GACATCCACCTTCCTCGTGTT; rat EB3: 

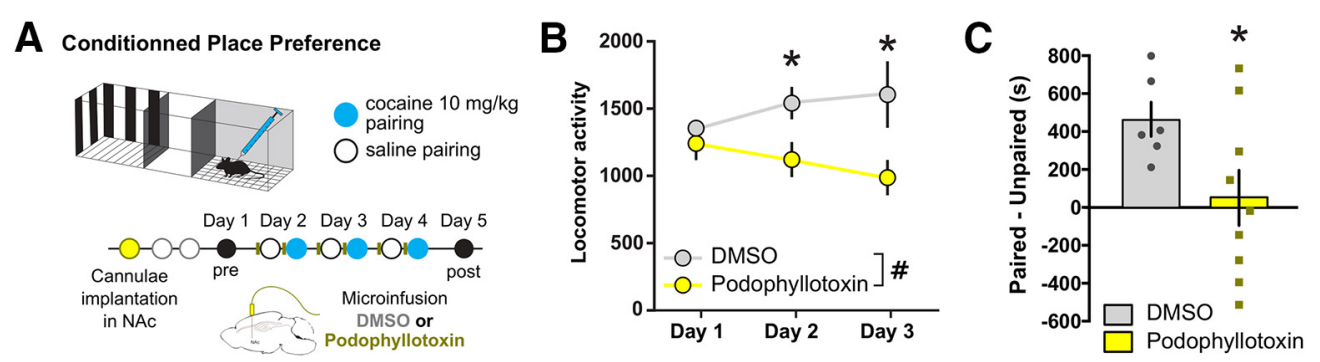

Figure 1. Inhibiting microtubule polymerization in NAc blocks cocaine-induced behavioral plasticity. $A$, Experimental time line. Mice were surgically implanted with microinfusion cannula, and the microtubule polymerization inhibitor podophyllotoxin $(n=6)$ or DMSO vehicle $(n=9)$ was infused into the NAc 30 min before cocaine conditioning with a 10 mg/kg dose. On the test day, preference was measured as the difference in time spent between the cocaine-paired and the saline-paired chambers. $B$, Locomotor sensitization to cocaine was blocked in mice treated with intra-NAc podophyllotoxin (two-way ANOVA with repeated measures; main effect of Day: $F_{(2,26)}=0.6054, p=0.5534$; main effect of Treatment: $F_{(1,13)}=6.185, p=0.0273$; interaction: $F_{(2,26)}$ $=2.406, p=0.1100$; followed by Holm-Sidak post hoc testing: day $1, p=0.8269 ;$ day $2, p=0.0415$; day $3, p=0.0415$ vs DMSO). C, Place preference formation was abolished by intra-NAc podophyllotoxin infusion (unpaired $t$ test: $t_{(13)}=2.182, p=0.0480$ ). Data are represented as the mean \pm SEM. \#p $<0.05$ for ANOVA main effects, ${ }^{*} p<0.05$ for $t$ tests and $p$ ost hoc tests.

forward, CCACCTCCTAACCCAGGTGAT; reverse, GGGGACGTC CTCTGTGGAAC; and rat SRCIN1: forward, CATGGGCATGCTTA AGTCGC; reverse, ACCATCTCTCTCCGGAGGTC.

Statistical analysis. Pairwise comparisons were performed using a twotailed unpaired Student's $t$ test with Welch's correction when appropriate. One-factor comparisons were performed using one-way ANOVA followed by Holm-Sidak post hoc tests or using a nonparametric Kruskal-Wallis test followed by Dunn's post hoc tests. The $2 \times 2$ comparisons were performed using two-way ANOVA with repeated measures for the appropriate factor and followed by Holm-Sidak post hoc tests. The $2 \times 2 \times 2$ comparisons were performed using three-way ANOVA with repeated measures for the appropriate factors and followed by Tukey's HSD post hoc tests. All statistical analyses were performed using GraphPad Prism, except for the three-way ANOVA that was performed using $\mathrm{R}$.

\section{Results}

Microtubule dynamics in the NAc regulate cocaine-induced behavioral plasticity

We first tested the possible involvement of microtubule polymerization in drug-related reward learning using an unbiased conditioned place preference assay in mice. This assay measures the ability of an animal to form an association between contextual cues and the rewarding effects of cocaine, and is typically used as an indirect measure of reward (Huston et al., 2013). To causally link microtubule remodeling to cocaine reward learning, the microtubule polymerization inhibitor podophyllotoxin (Desbène and Giorgi-Renault, 2002) or a control DMSO vehicle was infused into the NAc using microinfusion cannulae $30 \mathrm{~min}$ before the first cocaine or saline injection (Fig. 1A). Across the conditioning sessions, locomotor activity in response to a cocaine injection (10 mg/kg, i.p.) was monitored (Fig. 1B). Intra-NAc infusion of podophyllotoxin blocked the locomotor sensitization to cocaine (main effect of treatment: $F_{(1,13)}=6.185, p=0.0273$ ), and post hoc testing revealed that the cocaine-induced locomotor response was different at days $2(p=0.0415)$ and $3(p=0.0415)$, but not on the first day of treatment $(p=0.8269)$, suggesting that podophyllotoxin specifically affected the development of cocaine-induced behavioral plasticity over repeated injections and not the acute hyperlocomotor effect of a single drug dose. In contrast, repeated podophyllotoxin infusions had no effect on locomotor activity under saline conditions $(95.4 \pm 4.40 \%$ of DMSO controls; $n=8 ; p=0.2731$ ). Podophyllotoxin infusion also blocked the formation of a preference for the cocaine-paired side (Fig. $1 C ; t_{(13)}=2.182, p=0.0480$ ). These findings demonstrate that, similar to actin (Russo et al., 2010; Toda et al., 2010), microtubule dynamics are critical to cocaine-induced behavioral plasticity and reward learning.

\section{SRC phosphorylation in the NAc is induced at the synapse} after early withdrawal from cocaine self-administration Our next goal was to identify which proteins might regulate microtubule remodeling following cocaine exposure using a longaccess self-administration model in rats (Fig. 2A). Before withdrawal, rats exhibited robust and stable cocaine selfadministration behavior for $10 \mathrm{~d}$ (Fig. 2B). Because molecular (Wolf, 2016), physiological (Huang et al., 2015), and structural (Russo et al., 2010; Gipson et al., 2014) alterations induced by cocaine follow specific temporal patterns, we analyzed synaptic protein regulation after either 1 or $30 \mathrm{~d}$ of withdrawal, a time frame during which drug-seeking incubates from low to high levels (Tran-Nguyen et al., 1998; Grimm et al., 2001). We performed subcellular fractionation from NAc samples to isolate the synaptoneurosomal compartment, enriched in synaptic content, before probing for proteins of interest. We focused on EB3 and SRC because they have been shown to mediate microtubule-actin cross talk necessary for structural synaptic plasticity in other brain regions (Jaworski et al., 2009; Repetto et al., 2014), and because SRC phosphorylation responds to experimenteradministered chronic cocaine exposure (Damez-Werno et al., 2016). After $1 \mathrm{~d}$ of withdrawal (Fig. $2 C)$, both $\operatorname{EB} 3\left(t_{(9.432)}=\right.$ 0.4037, $p=0.6954)$ and total SRC $\left(t_{(16)}=1.517, p=0.1488\right)$ levels were unaffected. However, the phosphorylation ratio (phospho/total) for phospho-SRC/FYN at its active Y416/420 site (Okada, 2012) — available antibodies cannot distinguish between phospho-SRC and phospho-FYN, which also show similar $M_{\mathrm{r}}$ values on Western blotting-was significantly increased by cocaine self-administration $\left(t_{(16)}=2.297, p=0.0354\right)$. Levels of phospho-SRC/FYN are also elevated significantly when normalized to actin $\left(t_{(16)}=2.152, p=0.0470\right.$; Fig. $\left.2 C\right)$. These data suggest that SRC kinase activity might regulate actin polymerization at that time point, as prior work suggests that SRC inhibits actin remodeling (Repetto et al., 2014).

\section{EB3 synaptic levels in the NAc are increased after withdrawal} from cocaine self-administration

After $30 \mathrm{~d}$ of withdrawal, the synaptic protein landscape was different (Fig. 2D), with increased levels of EB3 $\left(t_{(12)}=2.268, p=\right.$ $0.0426)$, total SRC $\left(t_{(12)}=2.719, p=0.0187\right)$, and phospho-SRC/ FYN-Y416/420 $\left(t_{(12)}=2.180, p=0.0498\right)$ when normalized to actin, but no change in phospho-SRC/FYN Y416/420 ratio (phospho/total; $t_{(12)}=0.5383, p=0.6002$ ), between cocaine and saline samples. The sustained increase in levels of phospho-SRC/ FYN (normalized to actin) could be explained by the observed decrease in SRCIN1 levels at this time point $\left(_{(12)}=2.61, p=\right.$ 
A
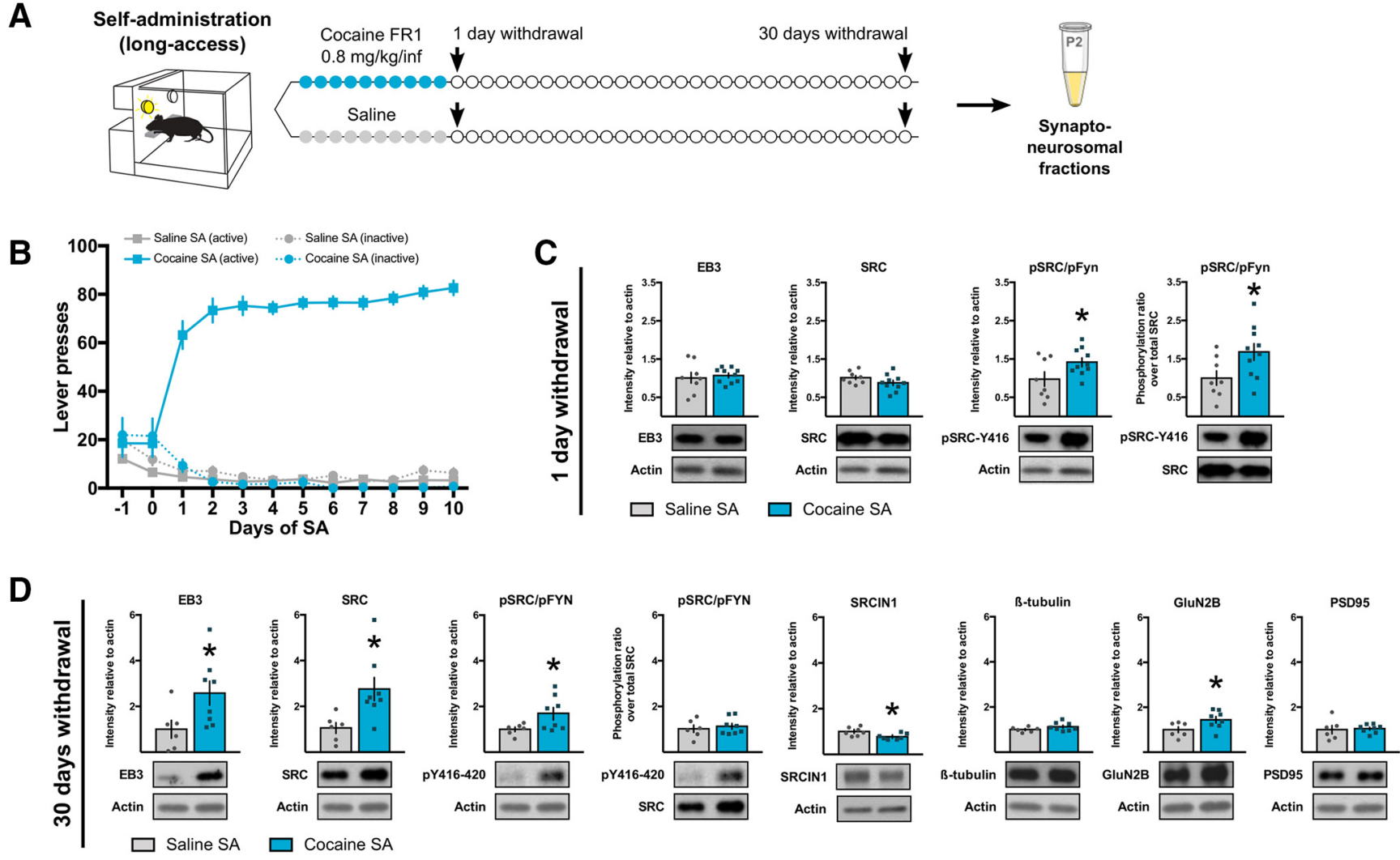

Figure 2. Synaptic EB3 levels and SRC phosphorylation are elevated in the NAc after withdrawal from cocaine-self administration (SA). $A$, Experimental time line. Rats were trained to self-administer cocaine or saline on an FR1 schedule of reinforcement for 10 consecutive days, and NAc tissue was collected after either $1 \mathrm{~d}(n=8$ saline, 10 cocaine) or $30 \mathrm{~d}$ ( $n=6$ saline, 8 cocaine) of withdrawal for subcellular fractionation. $\boldsymbol{B}$, Lever-pressing behavior across the 10 self-administration sessions. $\boldsymbol{C}$, Western blot quantification (top) and representative images (bottom) showing increased SRC/FYN phosphorylation at Y416/420 after $1 \mathrm{~d}$ of withdrawal when normalized to either actin (unpaired $t$ test: $\left.t_{(16)}=2.152, p=0.0470\right)$ or total SRC levels (unpaired $t$ test: $t_{(16)}=2.297, p=0.0354$ ) but no change in EB3 levels (unpaired Welch's $t$ test; $t_{(9.432)}=0.4037, p=0.6954$ ) and total SRC levels (unpaired $t$ test: $t_{(16)}=$ $1.517, p=0.1488$ ). $D$, Western blot quantification (top) and representative images (bottom) showing increased EB3 (unpaired $t$ test; $t_{(12)}=2.268, p=0.0426$ ), GluN2B (unpaired $t$ test; $t_{(12)}=2.299, p=0.0482$ ) and total SRC (unpaired $t$ test $t_{(12)}=2.719, p=0.0187$ ) levels after $30 \mathrm{~d}$ of withdrawal. SRC/FYN phosphorylation was increased when compared with actin levels (unpaired $t$ test: $t_{(12)}=2.180, p=0.0498$ ), but not when compared with total SRC (unpaired $t$ test: $\left.t_{(12)}=0.5383, p=0.6002\right)$. SRCIN1 levels were slightly decreased (unpaired $t$ test: $t_{(12)}=2.61, p=0.0228$ ). $\beta$-Tubulin (unpaired $t$ test: $t_{(12)}=1493, p=0.1612$ ) and PSD-95 (unpaired Welch's $t$ test: $t_{(6.302)}=0.2346, p=0.8220$ ) levels were similar between groups. Data are represented as the mean \pm SEM. ${ }^{*} p<0.05$ for $t$ tests.

0.0228; Fig. 2D), since SRCIN1 has been shown to be an indirect and selective inhibitor of SRC Y416 phosphorylation (Di Stefano et al., 2007). Expression of the glutamate ionotropic receptor subunit, GluN2B, a marker of spine stability (Akashi et al., 2009) known to participate in cocaine-induced synaptic plasticity (Ortinski, 2014), was also increased after $30 \mathrm{~d}$ of withdrawal. By contrast, levels of the microtubule building block $\beta$-tubulin $\left(t_{(12)}=\right.$ $1493, p=0.1612$ ), as well as the postsynaptic density scaffolding protein PSD-95 $\left(t_{(6.302)}=0.2346, p=0.8220\right)$, remained unaffected by cocaine self-administration. Together, these findings support the idea that changes in protein expression and signaling at NAc synapses occur in successive waves during the withdrawal period, and could be a biochemical substrate to explain the different structural (Russo et al., 2010; Gipson et al., 2014), physiological (Huang et al., 2015), and behavioral (Tran-Nguyen et al., 1998; Grimm et al., 2001; Wolf, 2016) phenotypes observed at different withdrawal time points. They also highlight SRC activation and EB3 as strong candidates to contribute to these adaptations during early versus late withdrawal.

\section{SRC activity in the NAc during early withdrawal is necessary for relapse}

To investigate the behavioral correlates of the protein expression changes that we observed during withdrawal from cocaine self- administration (Fig. 2), we took advantage of HSV-mediated overexpression strategies to specifically manipulate SRC activity and EB3 levels before behavioral testing. HSVs express their transgenes-in neurons selectively-at maximal levels between $12 \mathrm{~h}$ and $4 \mathrm{~d}$ after injection, with expression dissipating completely by day 7 (Neve et al., 2005). First, we explored the causal role of SRC activation during early withdrawal. After $10 \mathrm{~d}$ of cocaine self-administration, rats were injected into the NAc with an HSV overexpressing SRCIN1 or a control GFP-expressing vector (Fig. 3A). Overexpression of SRCIN1 mRNA at physiologically relevant levels - an approximately twofold increase-was confirmed by real-time PCR on rat NAc tissue collected $4 \mathrm{~d}$ after HSV infusion (Fig. $3 B ; p=0.0458$ vs HSV-GFP). The use of SRCIN1 is ideal, since it mediates cross talk between EB3-bound microtubules and activity-dependent structural plasticity via SRC regulation in vitro (Jaworski et al., 2009; Repetto et al., 2014), and is induced in the NAc following chronic intraperitoneal cocaine administration and opposes certain cocaine-related behaviors (Damez-Werno et al., 2016). There was no bias in selfadministration behavior before assignment to either group, as shown in Figure $3 D$, indicating that groups were appropriately counterbalanced. Because HSV expression is transient, SRC phosphorylation was inhibited only during the early phase of withdrawal when drug seeking was tested for the first time (at day 
A

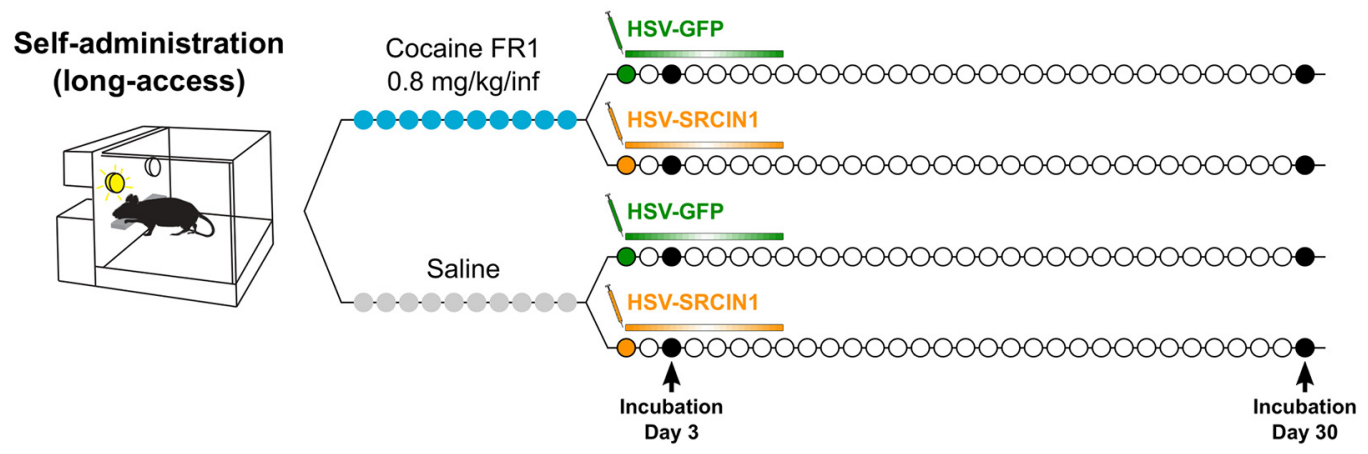

B

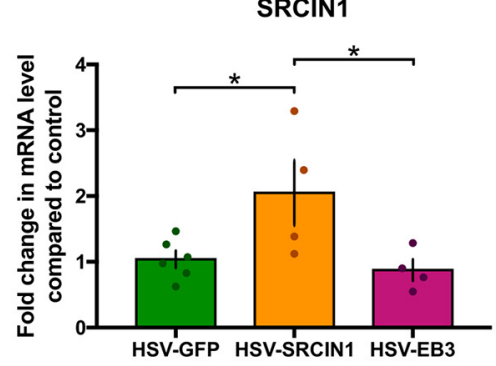

D

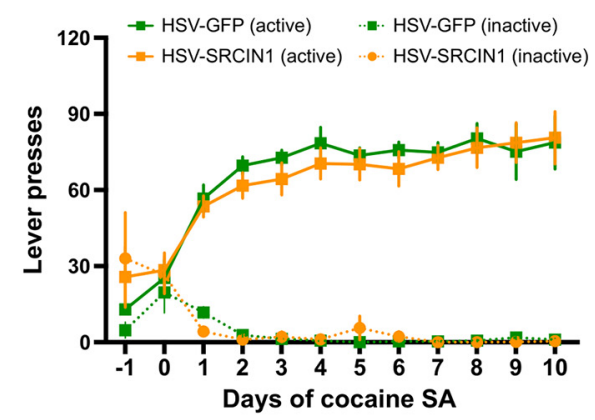

C

EB3

C

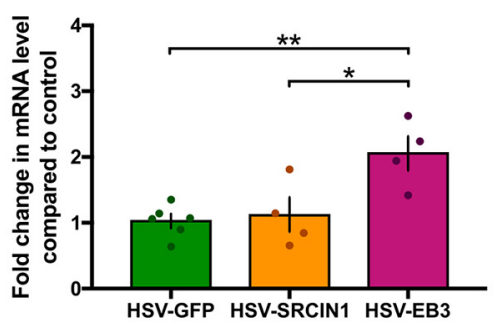

E

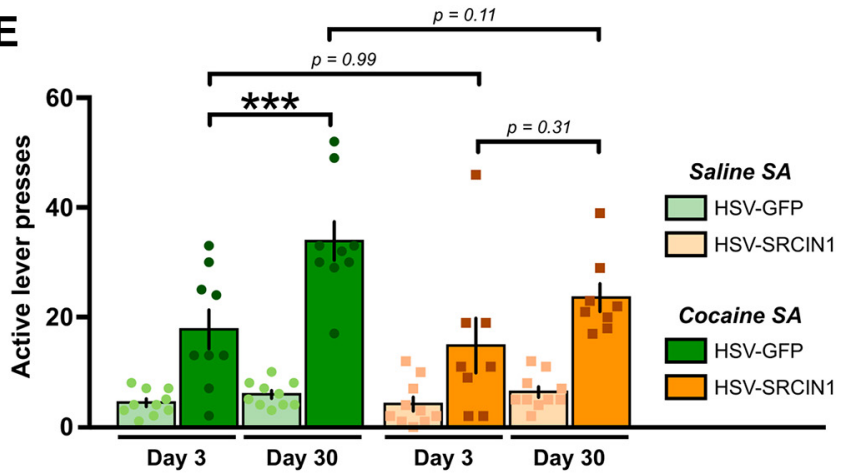

Figure 3. SRCIN1 overexpression in the NAc diminishes incubation of cocaine craving. $\boldsymbol{A}$, Experimental time line. Rats were trained to self-administer cocaine or saline on a FR1 schedule of reinforcement for 10 consecutive days before being injected in the NAc with an HSV overexpressing either SRCIN1, a negative regulator of SRC phosphorylation, or a control GFP vector ( $n=10$ saline-GFP, $n=10$ saline-SRCIN1, $n=9$ cocaine-GFP, $n=8$ cocaine-SRCIN1). Seeking was then measured after 3 and $30 \mathrm{~d}$ of withdrawal. $\boldsymbol{B}, 0$ verexpression of SRCIN1 in the NAc of rats $4 \mathrm{~d}$ after HSV-SRCIN1 infusion in this brain region was confirmed by real-time $P C R$, with no effect on the SRCIN1 expression seen with HSV-EB3 infusion (one-way ANOVA; $F_{(2.11)}=5.003, p=0.0285$; followed by Holm-Sidak post hoc testing: HSV-SRCIN1: $p=0.0458$ vs HSV-GFP; $p=0.0458$ vs HSV-EB3. C, Conversely, the overexpression of EB3 in the NAc of rats $4 \mathrm{~d}$ after HSV-EB3 infusion in this brain region was confirmed by real-time $P C R$, with no effect on the EB3 expression seen with HSV-SRCIN1 (one-way ANOVA: $F_{(2,11)}=8.515, p=0.0058$; followed by Holm-Sidak post hoc testing: HSV-EB3: $p=0.0074$ vs HSV-GFP; $p=0.0151$ vs HSV-SRCIN1. $\boldsymbol{D}$, Lever pressing across the 10 self-administration (SA) sessions showing no difference in intake before HSV group assignment. $\boldsymbol{E}$, SRCIN1 transient overexpression in the NAc during early withdrawal diminished incubation at a later time point (three-way ANOVA with repeated measures; main effect of Drug: $F_{(1,33)}=131.860$, $p<0.0001$; main effect of HSV: $F_{(1,33)}=3.731, p=0.0621$; main effect of Day: $F_{(1,33)}=11.354, p=0.00193$; interaction Drug $\times$ HSV: $F_{(1,33)}=4.865, p=0.0345$; interaction Drug $\times$ Day: $F_{(1,33)}=7.087, p=0.0119$; interaction HSV $\times$ Day: $F_{(1,33)}=0.549, p=0.464$; interaction Drug $\times$ HSV $\times$ Day: $F_{(1,33)}=0.986, p=0.3280 ;$ followed by Tukey HSD post hoc testing: CocaineSA. GFP.Day30 vs Day3, $p<0.001$; CocaineSA.SRCIN1.Day30 vs Day3, $p=0.3069$; CocaineSA.Day3.SRCIN1 vs GFP, $p=0.9943$; CocaineSA.Day30.SRCIN1 vs GFP, $p=0.1089$ ). Data are represented as the mean \pm SEM. ${ }^{*} p<0.05,{ }^{* *} p<0.01,{ }^{* * *} p<0.001$ for post hoc tests.

3 of withdrawal), but not for a second time after $30 \mathrm{~d}$ of withdrawal to measure incubation. We found that cocaine selfadministering HSV-SRCIN1-injected animals failed to exhibit incubation of cocaine seeking when comparing the levels of leverpressing behavior in the absence of drug between the first and second test days (Fig. $3 E ; p=0.3069$ ), whereas incubation was significant in HSV-GFP controls $(p<0.001)$. Interestingly however, seeking behavior was not different between HSV-SRCIN1injected and HSV-GFP-injected rats at day $3(p=0.9943)$, when SRCIN1 is in fact overexpressed and SRC phosphorylation inhibited. These results suggest that SRC activity in the NAc does not control drug-seeking behavior per se, but rather is necessary to set the stage for plastic changes responsible for incubation of drug seeking, which become apparent later. It also further confirms earlier findings that SRCIN1 is crucial to cocaine-induced behavioral adaptations (Damez-Werno et al., 2016), and might act upstream of SRC to coordinate structural plasticity in the NAc.

\section{EB3 in the NAc enhances motivation and drug seeking}

The next step was to study the behavioral consequences of the EB3 upregulation observed in the NAc after $30 \mathrm{~d}$ of withdrawal, with the hypothesis that this change might contribute to drug seeking and relapse. To this end, we combined HSV-mediated overexpression of EB3 in the NAc with a threshold procedure that allows for a behavioral economics analysis of consummatory and appetitive responding independently (Fig. 4A; Oleson and Roberts, 2009). Overexpression of EB3 mRNA at physiologically relevant levels - an approximately twofold increase-was con- 
A
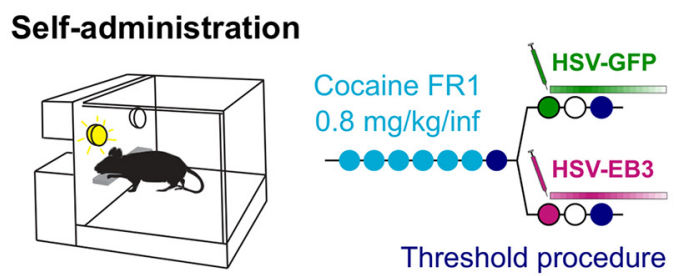

Threshold procedure
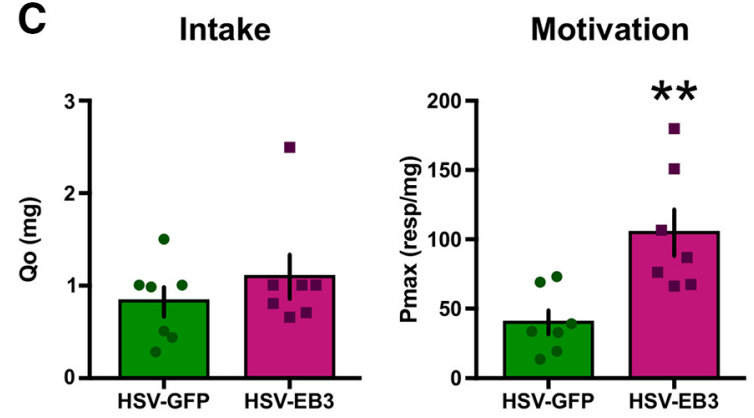

B

Dose-response curve

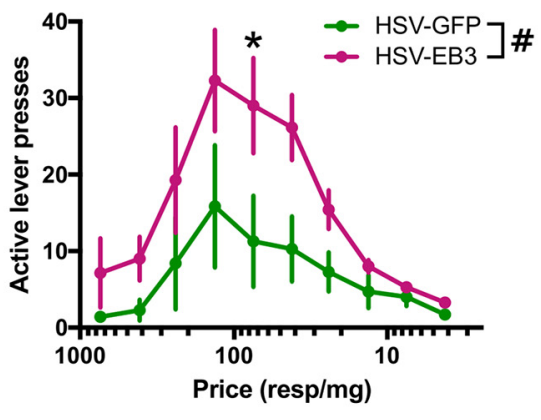

Elasticity

Perseverative responding
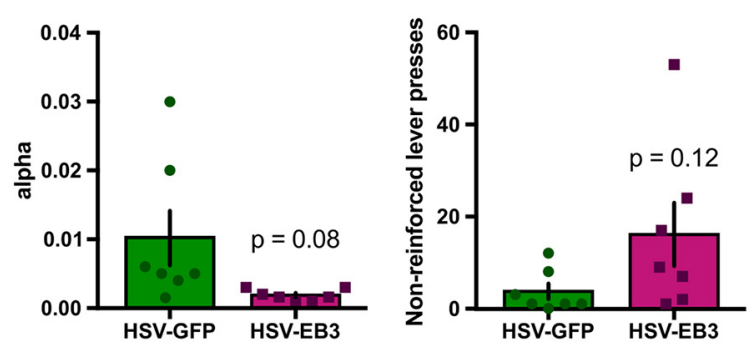

Figure 4. EB3 overexpression in the NAc increases motivation. $A$, Experimental time line for behavioral economics testing. Rats were trained to self-administer cocaine or saline on an FR1 schedule of reinforcement before being injected into the NAc with an HSV overexpressing either EB3 $(n=7)$ or a control GFP vector $(n=7)$, and subsequently went through the threshold procedure. $\boldsymbol{B}$, Dose-response curves showing that EB3 overexpression increased responding for cocaine [two-way ANOVA with repeated measures; main effect of Price: $F_{(9,108)}=10.38, p<0.0001 ;$ main effect of HSV: $F_{(1,12)}=4.982, p=0.0454$; interaction: $F_{(9,108)}=1713, p=0.0947$; followed by Holm-Sidak post hoc testing: price 42.1 responses $($ resp $) / \mathrm{mg}, p=0.0689 ;$ price 75 resp $/ \mathrm{mg}, p=0.0353$;

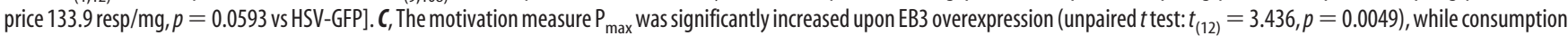
remained unaffected (unpaired $t$ test: $t_{(12)}=0.9662, p=0.35$ ). The elasticity parameter $\alpha$ was trending toward a decrease (unpaired Welch's test: $t_{(6.075)}=2.073, p=0.0830$ ), and perseverative responding trended toward an increase (unpaired Welch's $t$ test: $t_{(6.743)}=1.754, p=0.1245$ ). Data are represented as the mean $\pm \mathrm{SEM}$. $\# p<0.05$ for ANOVA main effects, ${ }^{*} p<0.05,{ }^{* *} p<0.01$ for $t$ tests and post hoc tests.

firmed by real-time PCR on rat NAc tissue collected $4 \mathrm{~d}$ after HSV infusion (Fig. $3 C ; p=0.0074$ vs HSV-GFP). To map the changes induced by EB3 overexpression onto complex motivated behaviors, we used a within-session threshold procedure, which allows for an entire dose-response curve to be generated within subject in a single day. In this task, the drug dose is reduced every $10 \mathrm{~min}$, which requires animals to increase response rates to maintain a constant level of drug intake. Thus, price (measured as the number of lever presses required to obtain $1 \mathrm{mg}$ of cocaine) is inversely related to drug dose (Fig. $4 B$ ). HSV-EB3 rats increased the number of responses across the dose-response curve compared with HSV-GFP controls (main effect of HSV: $F_{(1,12)}=4.982, p=$ $0.0454)$. Post hoc testing confirmed that this effect was significant for a 75 responses/mg price $(p=0.0353)$, and was almost significant at 42.1 responses/mg $(p=0.0689)$ and 133.9 responses $/ \mathrm{mg}$ $(p=0.0593)$. Next, data were plotted as a demand curve: individual consumption levels (in milligrams of cocaine) as a function of price. Basic economic principles were then applied through mathematical modeling to dissociate measures of motivation, consumption, and perseverative responding (Oleson and Roberts, 2009; Siciliano and Jones, 2017). Generally, when the dose is relatively high (i.e., price relatively low), the animal will maintain its preferred drug consumption level, termed $Q_{0}$. As price is increased, a point is eventually reached where animals will no longer maintain preferred intake levels. This inflection point is referred to as $\mathrm{P}_{\max }$, the maximal price an animal is willing to pay to self-administer cocaine. Finally, the exponential slope $\alpha$ at which drug consumption decreases beyond $\mathrm{P}_{\max }$ measures the elasticity of the demand curve and can be understood as an in- versely correlated indicator of motivation (lower $\alpha$ values describe higher motivation). Figure $4 C$ compares these various parameters between HSV-GFP-injected and HSV-EB3-injected animals. EB3 overexpression had no effect on drug intake, as $Q_{0}$ was equivalent between groups $\left(t_{(12)}=0.9662, p=0.35\right)$; however, it increased $\mathrm{P}_{\max }\left(t_{(12)}=3.436, p=0.0049\right)$ and strongly trended toward decreasing $\alpha\left(t_{(6.075)}=2.073, p=0.0830\right)$. The doses delivered during the last two bins of the threshold procedure are extremely low (prices of 416 and 750 responses/mg) and are not reinforcing when presented in isolation. Previous work has shown that lever pressing during these final time bins represents perseverative responding (Siciliano and Jones, 2017). EB3 overexpression trended toward increasing the number of lever presses during these epochs $\left(t_{(6.743)}=1.754, p=0.1245\right)$. Together, these results demonstrate that mimicking the EB3 increase that is evident only after long withdrawal (Fig. 1D) is sufficient to drive the motivation to consume cocaine and perhaps increase seeking behavior. This is consistent with increased drug seeking at this time point (Tran-Nguyen et al., 1998; Grimm et al., 2001), and thus posits EB3 levels in the NAc as an important mediator of drug seeking. The ability of EB3 overexpression in NAc to increase motivation for, but not consumption of, cocaine is precedented, because motivation and consumption are mediated by partly distinct circuitry and molecular mechanisms and can undergo independent regulation (Richardson and Roberts, 1996; Bentzley et al., 2014; Siciliano and Jones, 2017). Furthermore, a mechanism that is selective for motivation may offer greater therapeutic potential since those that affect consumption 
A

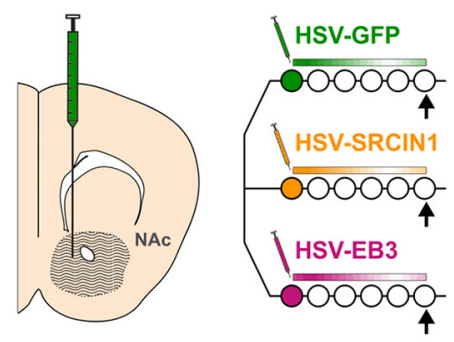

C

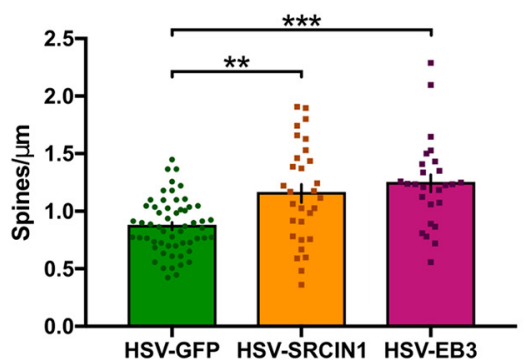

B

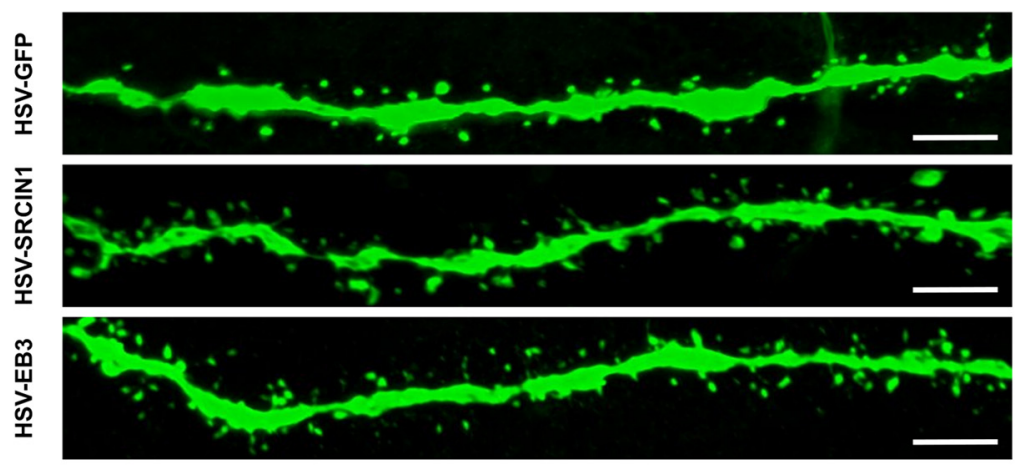

Thin spines

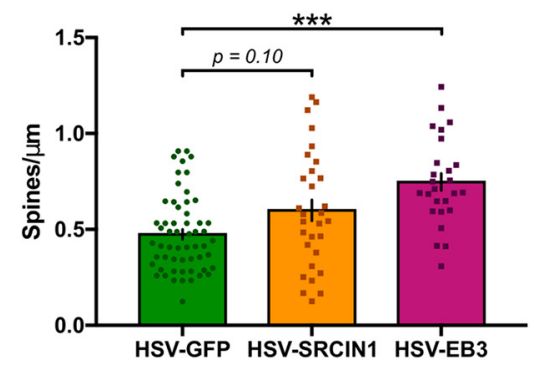

Mushroom spines

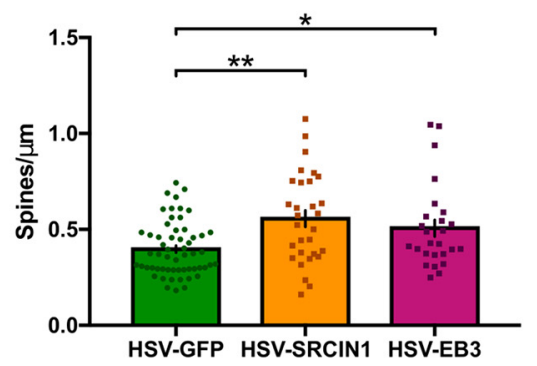

Figure 5. EB3 and SRCIN1 overexpression in the NAc both increase spine density and modulate dendritic spine morphology. $\boldsymbol{A}$, Experimental time line for spine morphology analysis. Mice were injected in the NAc with an HSV overexpressing EB3 ( $n=26$ neurites across 3 mice), SRCIN1 ( $n=30$ neurites across 3 mice), or a control GFP vector ( $n=57$ neurites across 6 mice), and were perfused for immunostaining after $5 \mathrm{~d}$. B, Representative confocal images of HSV-infected neurites. Scale bar, $5 \mu \mathrm{m}$. $\boldsymbol{C}$, Both HSV-SRCIN1 and HSV-EB3 viruses increased the total number of spines (Kruskal-Wallis test: $\mathrm{H}_{(2)}=22.53, p<0.0001$; followed by Dunn's post hoc testing: HSV-SRCIN1, $p=0.021$; HSV-EB3, $p<0.0001$ vs HSV-GFP), affecting both thin (Kruskal-Wallis test: $\mathrm{H}_{(2)}=20.46, p<0.0001$; followed by Dunn's post hoc testing: HSV-SRCIN1, $p=0.1016$; HSV-EB3, $p<0.0001$ vs HSV-GFP) and mushroom spines (Kruskal-Wallis test: $\mathrm{H}_{(2)}=12.78, p=0.0017$; followed by Dunn's post hoc testing: HSV-SRCIN1, $p=0.0016 ;$ HSV-EB3, $p=0.0465$ vs HSV-GFP). Data are represented as the mean \pm SEM. ${ }^{*} p<0.05,{ }^{* *} p<0.01,{ }^{* *} p<0.001$ for post hoc tests.

might be more likely to adversely influence the intake of natural rewards (Banks et al., 2019).

\section{EB3 and SRCIN1 control dendritic spine growth and} structural remodeling in the NAc

Because both EB3 (Jaworski et al., 2009) and SRCIN1 (Jaworski et al., 2009; Repetto et al., 2014) have been shown to regulate cytoskeletal dynamics and dendritic spine architecture in neurons cultured from other brain regions, we hypothesized that their behavioral effects (Figs. 3, 4) might be associated with structural changes to NAc neurons in vivo. To test this hypothesis, we assessed dendritic spine number and morphology after either EB3 or SRCIN1 overexpression in the NAc using the same HSV constructs used earlier (Fig. 5A). Representative confocal images of infected neurites are shown in Figure $5 B$. Quantification of the total number of spines per neurite (Fig. $5 C$ ) revealed that overexpression of either SRCIN1 $(p=0.021)$ or EB3 $(p<0.0001)$ increased total spine density in the NAc, suggesting that both have similar positive effects on spine growth. When further distinguishing between thin and mushroom spine morphology, which are thought to represent, respectively, more immature versus more mature forms of dendritic spines (Kasai et al., 2003), we showed that both spine types are affected. Thin spines were significantly increased by EB3 overexpression $(p<0.0001)$, and the same effect was close to significant after SRCIN1 overexpression $(p=0.1016)$. Mushroom spines were also increased by both viruses (SRCIN1, $p=0.0016$; EB3, $p=0.0465$ ), suggesting that EB3 and SRCIN1 are positive not only for spine growth but also for spine maturation and stabilization. These findings strengthen the idea that cocaine-induced increases in EB3 expression, or suppression of SRC phosphorylation by SRCIN1, in the NAC have the ability to establish similar structural plasticity in the NAc. Moreover, the ability of EB3 and SRCIN1 overexpression to induce mushroom spines on NAc medium spiny neurons is particularly noteworthy, since prior studies that manipulated synaptic actin dynamics alone in the NAc affected predominantly thin spines (Toda et al., 2010; Dietz et al., 2012).

Rapid changes in synaptic proteins in the NAc by a cocaine challenge after prolonged withdrawal

Persistent structural plasticity, while believed to encode enduring phenotypical changes, is nevertheless labile, as similar processes have been shown to be rapidly engaged after a subsequent drug or cue challenge (Gipson et al., 2014). To examine whether the same EB3-associated and SRC-associated pathways are also involved in such fast-reacting responses, we probed for the same target proteins as before (Fig. 2) in synaptoneurosomal fractions obtained from the NAc of cocaine or saline self-administering rats after $30 \mathrm{~d}$ of withdrawal killed $1 \mathrm{~h}$ after an $10 \mathrm{mg} / \mathrm{kg}$, i.p., cocaine challenge (Fig. 6A). After the challenge (Fig. $6 B)$, EB3 $\left(t_{(15)}=\right.$ $0.4937, p=0.6287)$, total SRC $\left(t_{(15)}=0.5111, p=0.6167\right)$, and SRCIN1 $\left(t_{(14)}=1.49, p=0.1583\right)$ levels were not statistically different between saline and cocaine self-administering animals, whereas phospho-SRC/FYN Y416/420 (normalized to total SRC) remained increased in rats with previous cocaine exposure compared with saline controls $\left(t_{(15)}=3.707, p=0.0021\right)$. This pattern is different from the synaptic protein expression pattern observed after prolonged withdrawal in the absence of a challenge 
A Self-administration
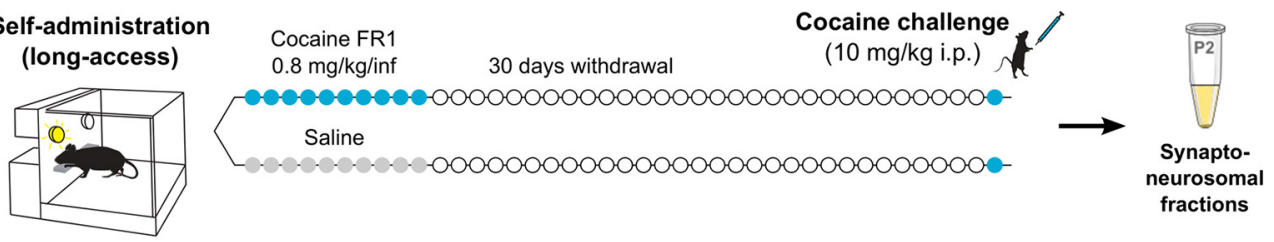

B
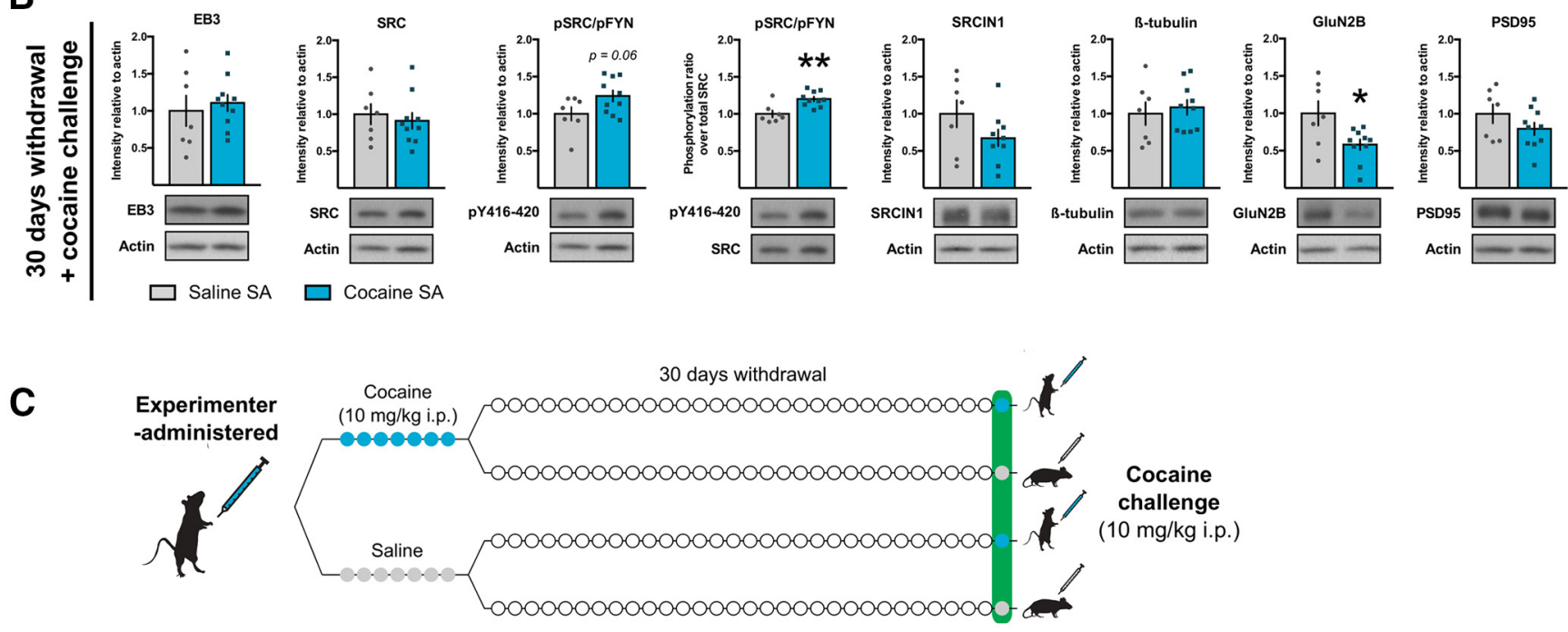

D
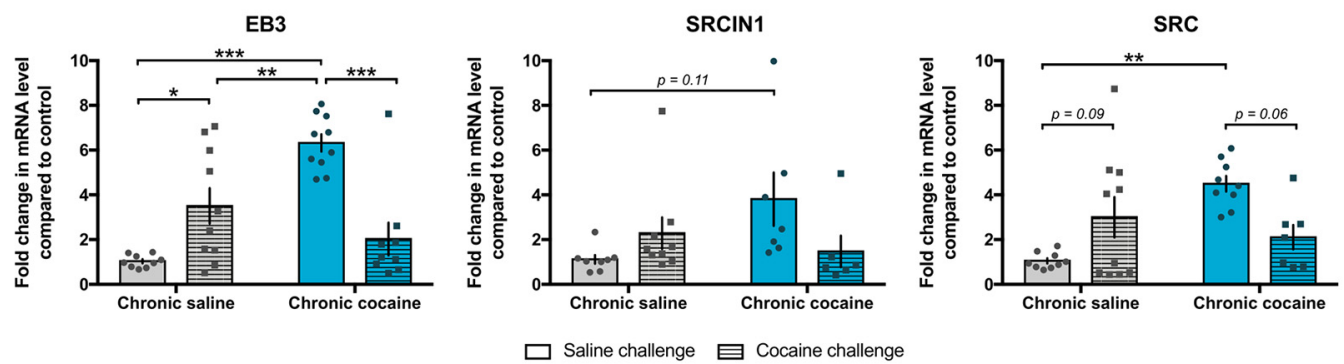

Figure 6. Cocaine challenge after prolonged withdrawal normalizes EB3 and total SRC levels in the NAc. $A$, Experimental time line. Rats were trained to self-administer cocaine $(n=10)$ or saline $(n=7)$ on an FR1 schedule of reinforcement for 10 consecutive days. After $30 \mathrm{~d}$ of withdrawal, animals were injected with an intraperitoneal $10 \mathrm{mg} / \mathrm{kg}$ dose of cocaine, and NActissue was collected $1 \mathrm{~h}$ later for subcellular fractionation. $\boldsymbol{B}$, Western blot quantification (top) and representative images (bottom) showing that the cocaine challenge increased SRC/FYN phosphorylation at $Y 416 / 420$ when normalized to total SRC levels (unpaired $t$ test: $t_{(15)}=3.707, p=0.0021$ ), with a trend seen when normalized to actin (unpaired $t$ test: $t_{(15)}=2.012, p=0.0626$ ), and decreased GluN2B levels (unpaired $t$ test: $t_{(15)}=2.617, p=0.0194$ ) in rats with previous cocaine self-administration (SA) experience compared with saline controls. Total EB3 (unpaired $t$ test: $t_{(15)}=0.4937, p=0.6287$ ), SRC (unpaired $t$ test: $\left.t_{(15)}=0.5111, p=0.6167\right), S R C I N 1$ (unpaired $t$ test: $t_{(14)}=1.49, p=0.1583$ ), $\beta$-tubulin (unpaired $t$ test: $t_{(15)}=0.4755, p=0.6413$ ), and PSD-95 (unpaired $t$ test: $t_{(15)}=$ $1.379, p=0.1881)$ levels, however, were not affected. C, Experimental time line. Mice were intraperitoneally injected with cocaine ( $10 \mathrm{mg} / \mathrm{kg})$ or saline daily for $7 \mathrm{~d}$, and with another cocaine (10 $\mathrm{mg} / \mathrm{kg}$ ) or saline challenge by intraperitoneal injection $30 \mathrm{~d}$ later ( $n=9$ saline $/ \mathrm{saline}, n=10$ saline/cocaine, $n=10$ cocaine $/ \mathrm{saline,} n=9$ cocaine/cocaine). D, Fold change in mRNA levels compared with saline/saline control for EB3 (two-way ANOVA with repeated measures; main effect of chronic treatment: $F_{(1,34)}=10.69, p=0.0025$; main effect of challenge: $F_{(1,34)}=2.435, p=$ 0.1279; interaction: $F_{(1,34)}=33.56, p<0.0001$; followed by Holm-Sidak post hoc testing: saline/cocaine vs saline/saline, $p=0.0153$; cocaine/saline vs saline/saline, $p<0.0001$; saline/cocaine vs cocaine/saline, $p=0.0051$; cocaine/saline vs cocaine/cocaine, $p<0.001$ ), SRCIN1 (two-way ANOVA with repeated measures; main effect of chronic treatment: $F_{(1,26)}=1.485, p=0.2340 ;$ main effect of challenge: $F_{(1,26)}=0.5752, p=0.4550$; interaction: $F_{(1,26)}=5.167, p=0.0315$; followed by Holm-Sidak posthoc testing: cocaine/saline vs saline/saline, $p=0.1110$ ) and SRC (two-way ANOVA with repeated measures; main effect of chronic treatment: $F_{(1,31)}=4.489, p=0.0422$; main effect of challenge: $F_{(1,31)}=0.1313, p=0.7195$; interaction: $F_{(1,31)}=13.05, p=0.0011$; followed by Holm-Sidak post hoc testing; saline/cocaine vs saline/saline, $p=0.0851$; cocaine/saline vs saline/saline, $p=0.0015$; cocaine/saline vs cocaine/cocaine, $p=0.0558$ ). Data are represented as the mean \pm SEM. ${ }^{*} p<0.05,{ }^{* *} p<0.01,{ }^{* * *} p<0.001$ for $t$ tests and post hoc tests.

(Fig. $2 D$ ), but is similar to that seen during early withdrawal (Fig. $2 C)$. $\beta$-Tubulin $\left(t_{(15)}=0.4755, p=0.6413\right)$ and PSD-95 $\left(t_{(15)}=\right.$ $1.379, p=0.1881$ ) remained unaffected, and GluN2B was decreased after the cocaine challenge in animals with a cocaine selfadministration history $\left(t_{(15)}=2.617, p=0.0194\right)$. The ability of EB3 or SRCIN1 overexpression to increase both thin and mushroom spines suggests that both proteins can promote de novo spine growth as well as spine maturation and enlargement. This is not surprising since mushrooms might arise not only from preexisting thin spines but also from newly formed spines. Moreover, because the HSV-mediated overexpression of the proteins cannot be targeted to individual spines that undergo morphological plasticity after drug exposure, such overexpression might force the emergence of new thin spines in addition to contributing to the stabilization of existing spines into mushroom spines. Together, these findings indicate that a cocaine challenge after prolonged withdrawal rapidly recapitulates at least part of the synaptic profile observed after early withdrawal. The results also suggest that some of the same molecular players-such as EB3, SRCIN1, and SRC - might be involved in the rapid mechanisms underlying the labile properties of drug-induced structural plasticity. 


\section{EB3, SRC, and SRCIN1 are transcriptionally regulated in the NAc by cocaine}

Finally, we studied whether cocaine regulation of EB3, SRCIN1, and SRC occur at the transcriptional level. To imitate the pharmacological effects of repeated cocaine exposure and parallel our previous experiments, mice were treated with intraperitoneal saline or cocaine for $10 \mathrm{~d}$, submitted to a saline or cocaine challenge $30 \mathrm{~d}$ later, and killed $1 \mathrm{~h}$ after (Fig. 6C). As depicted in Figure 6D, EB3 mRNA levels in the NAc were increased by a single, acute dose of cocaine (saline/cocaine vs saline/saline, $p=0.0153$ ), and by chronic cocaine and $30 \mathrm{~d}$ withdrawal (cocaine/saline vs saline/ saline, $p<0.0001$ ), but were normalized by the cocaine challenge in animals with previous cocaine exposure (cocaine/saline vs cocaine/cocaine, $p<0.001$ ). This suggests, first, that the normalization effect we observed at the protein level (Fig. $6 \mathrm{~B}$ ) could be due to the combination of a challenge-induced upregulation in saline self-administering animals and a downregulation in cocaine self-administering animals. The same interpretation can be made for total SRC levels, with SRC mRNA following a similar pattern as EB3 (Fig. 6C), with only the chronic cocaine effect reaching significance (cocaine/saline vs saline/saline, $p=$ 0.0015). SRCIN1 also seemed to follow the same regulation, without reaching significance, however. This trend in the regulation of SRCIN1 mRNA expression runs counter to the slight decrease in SRCIN1 protein levels observed after $30 \mathrm{~d}$ of withdrawal without a cocaine challenge (Fig. 2D), underscoring more complex regulation of this protein. In any event, we conclude that these three proteins are most likely transcriptionally regulated following drug exposure. Furthermore, because the expression of their mRNAs follows similar patterns of regulation, we speculate that this might be achieved through shared activity-dependent signaling pathways and upstream transcriptional regulators.

\section{Discussion}

In this study, we show that microtubule dynamics are important for cocaine-induced plasticity in the NAc and identify microtubule-bound EB3, SRCIN1, and SRC as important mediators of this process. Using a combination of viral approaches, we confirmed the causal role of these targets during withdrawal from cocaine self-administration in triggering relapse-like behaviors, effects that are associated with structural adaptations in dendritic architecture. Our data support the idea of cooperation between actin and microtubule dynamics specifically at NAc synapses in mediating activity-dependent but nevertheless persistent adaptations that underlie the maladaptive learning implicated in cocaine addiction.

The contribution of cytoskeletal dynamics to drug-induced adaptations is well established. At the structural level, chronic cocaine exposure is associated with changes in dendritic spine architecture (Robinson and Kolb, 2004; Russo et al., 2010; Gipson et al., 2014), for which actin polymerization is necessary (Toda et al., 2010). The current model posits that a subset of thin spines induced by cocaine mature over time into enlarged mushroom spines that encode stable drug-related memories (Gipson et al., 2014; Huang et al., 2015). Our result implicating microtubule polymerization in cocaine-induced plasticity (Fig. 1) is one of the first looking at cytoskeletal components other than actin. Consistent with a role for microtubules in the plastic changes underlying addiction in general, microtubule-related signaling has been implicated previously in alcohol abuse models (Liu et al., 2017). Drug-induced spine remodeling thus involves mechanisms more complex than previously assumed, requiring the coordination of actin and microtubules, but also of noncanonical cytoskeletal partners such as nonmuscle myosin II (Briggs et al., 2017), across various time scales.

Cross talk between actin filaments and microtubules has been proposed in other systems. Most of the supporting evidence, however, comes from in vitro studies of the axonal growth cone and suggests that actin-microtubule interactions result in the reciprocal stabilization of these otherwise dynamic structures (Coles and Bradke, 2015). A similar process might explain the time-dependent structural changes that dendritic spines undergo during plasticity (Kasai et al., 2003), with a first step of active reorganization followed by stabilization of the newly remodeled spines (Kasai et al., 2003; Hotulainen and Hoogenraad, 2010; Borovac et al., 2018). In vitro work demonstrated that the interaction between EB3 and SRCIN1, only possible upon invasion of growing EB3-bound microtubules into dendritic spines, alters spine morphology in cultured cortical and hippocampal neurons (Jaworski et al., 2009). EB3 and SRCIN1 are seemingly both positive for spine stabilization and maturation, because knockdown of either protein resulted in a similar phenotype, with decreased mushroom and increased thin spines (Jaworski et al., 2009; Repetto et al., 2014). In vivo, however, the dendritic spine phenotype in hippocampal neurons of SRCIN1 knock-out mice was not as clear (Repetto et al., 2014). This might be due to a non-cellautonomous developmental compensation mechanism, as our spine imaging data using SRCIN1 overexpression in NAc neurons revealed increased mushroom spines (Fig. 5), which is consistent with the in vitro findings (Jaworski et al., 2009). Moreover, the fact that we found similar dendritic spine phenotypes after EB3 and SRCIN1 overexpression (Fig. 5) suggests that both proteins act in concert to promote both spine growth (as evidenced by increased thin spines) and maturation (as evidenced by increased mushroom spines) in the NAc in vivo. Mechanistically, EB3 and SRCIN1 have been proposed to stabilize spines by promoting actin polymerization through a complex signaling pathway involving, among many other mechanisms, SRC phosphorylation (Repetto et al., 2014). SRC phosphorylation at the Y416 residue triggers signaling cascades, ultimately inhibiting actin polymerization (Repetto et al., 2014).

Following cocaine self-administration, we show that SRC phosphorylation was induced in the NAc during early withdrawal, while EB3 levels were increased only after longer withdrawal (Fig. 2). In light of the respective roles of SRC phosphorylation and EB3 regarding cytoskeleton and spine remodeling (see above), this two-step scenario could coincide with a two-step mechanism of spine restructuring and stabilization (Fig. 7). Early after drug exposure, when spines are putatively still undergoing restructuring, SRC phosphorylation is induced and would be expected to inhibit actin polymerization. Synaptic weakening caused by a deteriorating actin cytoskeleton at early withdrawal time points, associated with thin spine formation, has previously been reported (Shen et al., 2009; Russo et al., 2010). This first stage of remodeling is believed to be followed by a longer-lasting state of spine stabilization (Russo et al., 2010; Gipson et al., 2014), and we here propose that the entry of microtubule-bound EB3 into dendritic spines observed after prolonged withdrawal from cocaine exposure might be a positive trigger for the above-mentioned signaling pathways, leading to actin polymerization and spine stabilization. Moreover, because SRCIN1 overexpression-mediated SRC inhibition selectively during early withdrawal reduces later drug seeking (Fig. 3), we can hypothesize that this first stage of SRC-dependent synaptic weakening is required for longer-lasting pathological adaptations underlying relapse, maybe by preventing a second stage of EB3- 
Early withdrawal

"Memory formation"

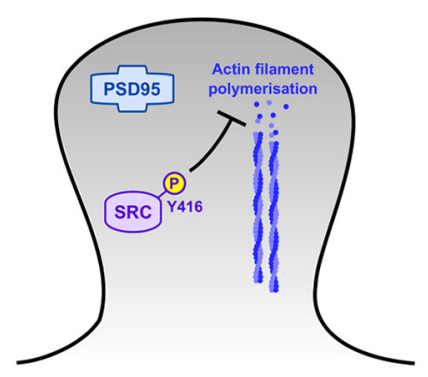

Actin depolymerisation
Thin spines
Long withdrawal

"Memory stabilization"

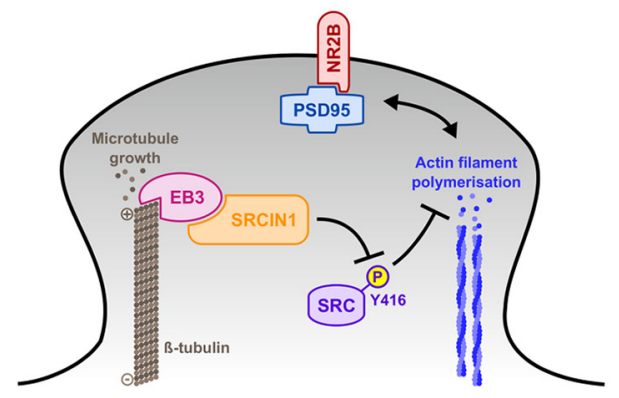

Microtubule invasion

Actin polymerisation Mushroom spines

\section{Cocaine challenge}

"Memory reconsolidation"

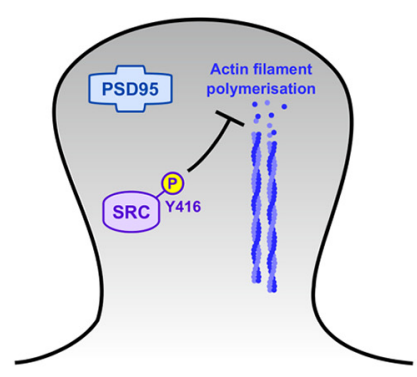

Actin depolymerisation
Thin spines

Figure 7. Proposed model of cooperation among EB3, SRCIN1, and SRC at NAc synapses regulating cytoskeletal dynamics and structural plasticity following cocaine self-administration. During early withdrawal from cocaine self-administration (left), SRC phosphorylation at its active Y416 site is induced at NAc synapses and indirectly inhibits actin polymerization. Actin cytoskeleton destabilization is associated with the emergence of thin, immature, and plastic spines during this stage of memory "formation." After prolonged withdrawal (center), EB3-bound dynamic microtubules have invaded NAC spines. Because it interacts with EB3, SRCIN1 (despite a slight reduction in total levels) is relocated in the postsynaptic compartment, where it inhibits SRC-Y416 phosphorylation and thus releases the inhibition of actin polymerization. This activation of actin polymerization creates enlarged mushroom spines associated with the "stabilization" of drug memories. After an acute cocaine challenge (right), EB3-bound and SRCIN1-bound microtubules exit NAC spines; SRC-Y416 phosphorylation is consequently induced and inhibits actin polymerization to revert mushroom spines into thin and labile spines, putatively allowing the drug memories to be "reconsolidated." This is a highly simplified scheme given the likely involvement of additional proteins and mechanisms in these phenomena.

mediated spine stabilization to occur, which we show increases the motivation to seek and consume cocaine (Fig. 4). This is also consistent with the published behavioral effects of SRCIN1 overexpression, where SRCIN1 opposed cocaine-induced behaviors (Damez-Werno et al., 2016). Because it may oppose the necessary first step of actin depolymerization controlled by SRC phosphorylation, SRCIN1 overexpression might impede the formation of drug-context associative memories that depend on structural plasticity, as well as reduce the reinforcing properties of cocaine (Damez-Werno et al., 2016). However, we find that SRCIN1 expression is slightly decreased at the $30 \mathrm{~d}$ withdrawal time point. This is consistent with increased levels of phospho-SRC/FYN (when normalized to actin, although not to total SRC) observed under these conditions, but counter to our hypothesis that prolonged withdrawal from cocaine self-administration is associated with suppression of SRC function by an EB3/SRCIN1-dependent mechanism. One explanation for these paradoxical observations is that sufficient levels of SRCIN1 remain after $30 \mathrm{~d}$ of withdrawal to interact with elevated levels of EB3 to suppress SRC function and enable the stabilization of mushroom-shaped dendritic spines. At the same time, these observations underscore the complexity of the biochemical mechanisms that control SRC function and spine dynamics in the NAc and suggest the involvement of still additional microtubule- and actin-associated proteins in these phenomena.

A key role for EB3 and SRC phosphorylation in mediating the cytoskeletal dynamics underlying spine morphological changes is further supported by our findings following a cocaine challenge after prolonged withdrawal (Fig. 6). Such a challenge rapidly reversed the elevated levels of EB3 and total SRC seen before the challenge dose of cocaine. These actions might reduce SRCIN1 inhibition of SRC and promote actin depolymerization, although additional proteins are likely involved, as noted above. This would fit with the current model according to which a cocaine challenge rapidly induces spine destabilization and the re- emergence of thin spines from mushroom spines (Shen et al., 2009; Russo et al., 2010; Huang et al., 2015; Spencer et al., 2017), and a similar mechanism could be at play when relapse is triggered by re-exposure to a drug-paired context (Stankeviciute et al., 2014). Overall, this mechanism could explain how supposedly stable mushroom spines remain labile to certain stimuli, and is consistent with the proposed mechanisms of memory reconsolidation (Sorg, 2012).

Another interesting finding was the regulation pattern of GluN2B. After $30 \mathrm{~d}$ of withdrawal from cocaine self-administration, synaptic expression of GluN2B was increased (Fig. 2), but was rapidly normalized by the cocaine challenge (Fig. 6). This seems discrepant with a previous report of unaffected synaptic GluN2B levels in the NAc after $45 \mathrm{~d}$ of withdrawal from cocaine self-administration (Ferrario et al., 2012). However, we cannot exclude that its regulation might be time sensitive, similar to GluN2B expression in prefrontal cortex, which increased specifically after $14 \mathrm{~d}$, but not after 1 or $60 \mathrm{~d}$ of withdrawal (Ben-Shahar et al., 2009). Functionally, GluN2B is involved in activitydependent plasticity, as it is important for the strengthening and stabilization of cocaine-induced new synapses (Lee and Dong, 2011), is regulated through SRC signaling (Sinai et al., 2010), and might act upstream of cytoskeleton remodeling, because GluN2B deletion causes a decrease in actin polymerization and spine density (Akashi et al., 2009). This could explain why GluN2B is increased after prolonged withdrawal, when mature spines are present, and is quickly downregulated by a cocaine challenge that is associated with spine weakening (Fig. 7).

In conclusion, this work represents an important advance in the study of cocaine-induced structural plasticity mechanisms, because it posits microtubules and associated signaling proteins as key mediators of spine remodeling and subsequent behavioral alterations. Further work is still needed to elucidate the precise temporal dynamics of microtubule remodeling and its coordination with actin remodeling following drug exposure, and to ob- 
tain a more complete view of the complex, time-dependent regulation of a host of actin- and microtubule-associated proteins that are likely involved in spine dynamics. A better understanding of how reward learning is encoded by dendritic spine changes at different time scales, from the first drug exposure to relapse even long into the withdrawal period, along with the identification of specific molecules engaged in the process, remains an important effort from both fundamental and translational standpoints.

\section{References}

Akashi K, Kakizaki T, Kamiya H, Fukaya M, Yamasaki M, Abe M, Natsume R, Watanabe M, Sakimura K (2009) NMDA receptor GluN2B (GluRe2/ NR2B) subunit is crucial for channel function, postsynaptic macromolecular organization, and actin cytoskeleton at hippocampal CA3 synapses. J Neurosci 29:10869-10882.

Banks ML, Townsend EA, Negus SS (2019) Testing the 10 most wanted: a preclinical algorithm to screen candidate opioid use disorder medications. Neuropsychopharmacology 44:1011-1012.

Ben-Shahar O, Obara I, Ary AW, Ma N, Mangiardi MA, Medina RL, Szumlinski KK (2009) Extended daily access to cocaine results in distinct alterations in homer $1 \mathrm{~b} / \mathrm{c}$ and NMDA receptor subunit expression within the medial prefrontal cortex. Synapse 63:598-609.

Bentzley BS, Jhou TC, Aston-Jones G (2014) Economic demand predicts addiction-like behavior and therapeutic efficacy of oxytocin in the rat. Proc Natl Acad Sci U S A 111:11822-11827.

Borovac J, Bosch M, Okamoto K (2018) Regulation of actin dynamics during structural plasticity of dendritic spines: signaling messengers and actin-binding proteins. Mol Cell Neurosci 91:122-130.

Briggs SB, Blouin AM, Young EJ, Rumbaugh G, Miller CA (2017) Memory disrupting effects of nonmuscle myosin II inhibition depend on the class of abused drug and brain region. Learn Mem 24:70-75.

Cahill ME, Bagot RC, Gancarz AM, Walker DM, Sun H, Wang ZJ, Heller EA, Feng J, Kennedy PJ, Koo JW, Cates HM, Neve RL, Shen L, Dietz DM, Nestler EJ (2016) Bidirectional synaptic structural plasticity after chronic cocaine administration occurs through Rap1 small GTPase signaling. Neuron 89:566-582.

Calipari ES, Godino A, Peck EG, Salery M, Mervosh NL, Landry JA, Russo SJ, Hurd YL, Nestler EJ, Kiraly DD (2018) Granulocyte-colony stimulating factor controls neural and behavioral plasticity in response to cocaine. Nat Commun 9:9.

Coles CH, Bradke F (2015) Coordinating neuronal actin-microtubule dynamics. Curr Biol 25:R677-R691.

Damez-Werno DM, Sun H, Scobie KN, Shao N, Rabkin J, Dias C, Calipari ES, Maze I, Pena CJ, Walker DM, Cahill ME, Chandra R, Gancarz A, Mouzon E, Landry JA, Cates H, Lobo MK, Dietz D, Allis CD, Guccione E, et al. (2016) Histone arginine methylation in cocaine action in the nucleus accumbens. Proc Natl Acad Sci U S A 113:9623-9628.

Day JJ, Carelli RM (2007) The nucleus accumbens and pavlovian reward learning. Neuroscientist 13:148-159.

Desbène S, Giorgi-Renault S (2002) Drugs that inhibit tubulin polymerization: the particular case of podophyllotoxin and analogues. Curr Med Chem Anti Inflamm Anti Allergy Agents 2:71-90.

Di Chiara G, Imperato A (1988) Drugs abused by humans preferentially increase synaptic dopamine concentrations in the mesolimbic system of freely moving rats. Proc Natl Acad Sci U S A 85:5274-5278.

Dietz DM, Sun H, Lobo MK, Cahill ME, Chadwick B, Gao V, Koo JW, MazeiRobison MS, Dias C, Maze I, Damez-Werno D, Dietz KC, Scobie KN, Ferguson D, Christoffel D, Ohnishi Y, Hodes GE, Zheng Y, Neve RL, Hahn KM, et al. (2012) Racl is essential in cocaine-induced structural plasticity of nucleus accumbens neurons. Nat Neurosci 15:891-896.

Di Stefano P, Damiano L, Cabodi S, Aramu S, Tordella L, Praduroux A, Piva R, Cavallo F, Forni G, Silengo L, Tarone G, Turco E, Defilippi P (2007) p140Cap protein suppresses tumour cell properties, regulating csk and src kinase activity. EMBO J 26:2843-2855.

Ferrario CR, Goussakov I, Stutzmann GE, Wolf ME (2012) Withdrawal from cocaine self-administration alters NMDA receptor-mediated Ca2+ entry in nucleus accumbens dendritic spines. PLoS One 7:e40898.

Geraldo S, Gordon-Weeks PR (2009) Cytoskeletal dynamics in growthcone steering. J Cell Sci 122:3595-3604.
Gipson CD, Kupchik YM, Kalivas PW (2014) Rapid, transient synaptic plasticity in addiction. Neuropharmacology 76:276-286.

Grimm JW, Hope BT, Wise RA, Shaham Y (2001) Neuroadaptation: incubation of cocaine craving after withdrawal. Nature 412:141-142.

Hotulainen P, Hoogenraad CC (2010) Actin in dendritic spines: connecting dynamics to function. J Cell Biol 189:619-629.

Huang YH, Schlüter OM, Dong Y (2015) Silent synapses speak up: updates of the neural rejuvenation hypothesis of drug addiction. Neuroscientist 21:451-459.

Hu X, Viesselmann C, Nam S, Merriam E, Dent EW (2008) Activitydependent dynamic microtubule invasion of dendritic spines. J Neurosci 28:13094-13105.

Hursh SR, Silberberg A (2008) Economic demand and essential value. Psychol Rev 115:186-198.

Huston JP, Silva MA, Topic B, Müller CP (2013) What's conditioned in conditioned place preference? Trends Pharmacol Sci 34:162-166.

Jaworski J, Kapitein LC, Gouveia SM, Dortland BR, Wulf PS, Grigoriev I, Camera P, Spangler SA, Di Stefano P, Demmers J, Krugers H, Defilippi P, Akhmanova A, Hoogenraad CC (2009) Dynamic microtubules regulate dendritic spine morphology and synaptic plasticity. Neuron 61:85-100.

Jordan MA, Thrower D, Wilson L (1992) Effects of vinblastine, podophyllotoxin and nocodazole on mitotic spindles. implications for the role of microtubule dynamics in mitosis. J Cell Sci 102:401-416.

Kasai H, Matsuzaki M, Noguchi J, Yasumatsu N, Nakahara H (2003) Structure-stability-function relationships of dendritic spines. Trends Neurosci 26:360-368.

Kelley AE, Berridge KC (2002) The neuroscience of natural rewards: relevance to addictive drugs. J Neurosci 22:3306-3311.

Koob GF, Volkow ND (2010) Neurocircuitry of addiction. Neuropsychopharmacology 35:217-238.

Krasnova IN, Marchant NJ, Ladenheim B, McCoy MT, Panlilio LV, Bossert JM, Shaham Y, Cadet JL (2014) Incubation of methamphetamine and palatable food craving after punishment-induced abstinence. Neuropsychopharmacology 39:2008-2016.

Lamprecht R, LeDoux J (2004) Structural plasticity and memory. Nat Rev Neurosci 5:45-54.

Lee BR, Dong Y (2011) Cocaine-induced metaplasticity in the nucleus accumbens: silent synapse and beyond. Neuropharmacology 61:10601069.

Liu F, Laguesse S, Legastelois R, Morisot N, Ben Hamida S, Ron D (2017) mTORC1-dependent translation of collapsin response mediator protein-2 drives neuroadaptations underlying excessive alcohol-drinking behaviors. Mol Psychiatry 22:89-101.

Neve RL, Neve KA, Nestler EJ, Carlezon WA Jr (2005) Use of herpes virus amplicon vectors to study brain disorders. Biotechniques 39:381-391.

Nimchinsky EA, Sabatini BL, Svoboda K (2002) Structure and function of dendritic spines. Annu Rev Physiol 64:313-353.

Okada M (2012) Regulation of the src family kinases by csk. Int J Biol Sci 8:1385-1397.

Oleson EB, Roberts DC (2009) Behavioral economic assessment of price and cocaine consumption following self-administration histories which produce escalation of either final ratios or intake. Neuropsychopharmacology 34:796-804.

Ortinski PI (2014) Cocaine-induced changes in NMDA receptor signaling. Mol Neurobiol 50:494-506.

Repetto D, Camera P, Melani R, Morello N, Russo I, Calcagno E, Tomasoni R, Bianchi F, Berto G, Giustetto M, Berardi N, Pizzorusso T, Matteoli M, Di Stefano P, Missler M, Turco E, Di Cunto F, Defilippi P (2014) p140Cap regulates memory and synaptic plasticity through src-mediated and citron-N-mediated actin reorganization. J Neurosci 34:1542-1553.

Richardson NR, Roberts DC (1996) Progressive ratio schedules in drug selfadministration studies in rats: a method to evaluate reinforcing efficacy. J Neurosci Methods 66:1-11.

Robinson TE, Kolb B (2004) Structural plasticity associated with exposure to drugs of abuse. Neuropharmacology 47:33-46.

Russo SJ, Dietz DM, Dumitriu D, Morrison JH, Malenka RC, Nestler EJ (2010) The addicted synapse: mechanisms of synaptic and structural plasticity in nucleus accumbens. Trends Neurosci 33:267-276.

Shen HW, Toda S, Moussawi K, Bouknight A, Zahm DS, Kalivas PW (2009) Altered dendritic spine plasticity in cocaine-withdrawn rats. J Neurosci 29:2876-2884.

Siciliano CA, Jones SR (2017) Cocaine potency at the dopamine transporter 
tracks discrete motivational states during cocaine self-administration. Neuropsychopharmacology 42:1893-1904.

Sinai L, Duffy S, Roder JC (2010) Src inhibition reduces NR2B surface expression and synaptic plasticity in the amygdala. Learn Mem 17:364-371.

Sorg BA (2012) Reconsolidation of drug memories. Neurosci Biobehav Rev 36:1400-1417.

Spencer S, Garcia-Keller C, Roberts-Wolfe D, Heinsbroek JA, Mulvaney M, Sorrell A, Kalivas PW (2017) Cocaine use reverses striatal plasticity produced during cocaine seeking. Biol Psychiatry 81:616-624.

Stankeviciute NM, Scofield MD, Kalivas PW, Gipson CD (2014) Rapid, transient potentiation of dendritic spines in context-induced relapse to cocaine seeking. Addict Biol 19:972-974.
Toda S, Shen H, Kalivas PW (2010) Inhibition of actin polymerization prevents cocaine-induced changes in spine morphology in the nucleus accumbens. Neurotox Res 18:410-415.

Tran-Nguyen LT, Fuchs RA, Coffey GP, Baker DA, O'Dell LE, Neisewander JL (1998) Time-dependent changes in cocaine-seeking behavior and extracellular dopamine levels in the amygdala during cocaine withdrawal. Neuropsychopharmacol Off Publ Am Coll Neuropsychopharmacol 19:48-59.

Volkow ND, Morales M (2015) The brain on drugs: from reward to addiction. Cell 162:712-725.

Wolf ME (2016) Synaptic mechanisms underlying persistent cocaine craving. Nat Rev Neurosci 17:351-365. 